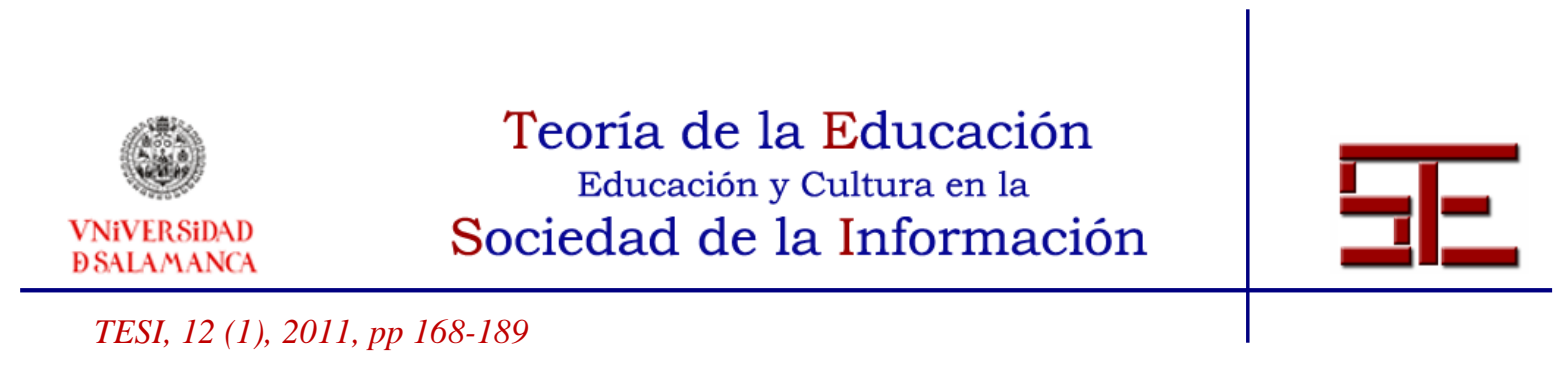

\title{
OPTIMIZACIÓN DEL COMPORTAMIENTO INFORMACIONAL MEDIANTE EL USO DE HERRAMIENTAS DE BOOKMARKING
}

Resumen: La actual sociedad del conocimiento así como las nuevas políticas educativas de la Unión Europea requieren una nueva aproximación a los recursos educativos. El uso de un formato cerrado y monolítico como puede ser el libro de texto tiene que ser substituido por la creación y actualización constante de colecciones o repositorios de recursos que faciliten y formen parte del proceso de aprendizaje. El presente trabajo introduce una aproximación a este nuevo paradigma a través del análisis de tres niveles: la investigación del comportamiento informacional de estudiantes en el uso de recursos educativos disponibles en la Red; el análisis funcional y de usabilidad de las herramientas que dan soporte a la gestión y uso de los recursos de información; y la prueba piloto de una herramienta de bookmarking en las aulas virtuales de la asignatura de Interacción Persona-Ordenador como práctica para mejorar el comportamiento informacional de los estudiantes. Considerar conjuntamente estos tres niveles es clave para mejorar el comportamiento informacional de los estudiantes $\mathrm{y}$, en consecuencia, permitir una mejor adquisición de las competencias informacionales esenciales en el contexto actual.

Palabras clave: herramientas docentes; etiquetado de recursos educativos; bookmarking; experiencia de usuario; competencias informacionales.

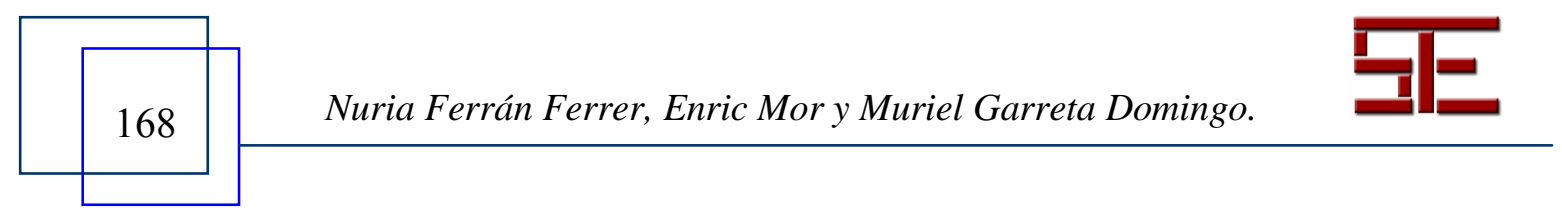




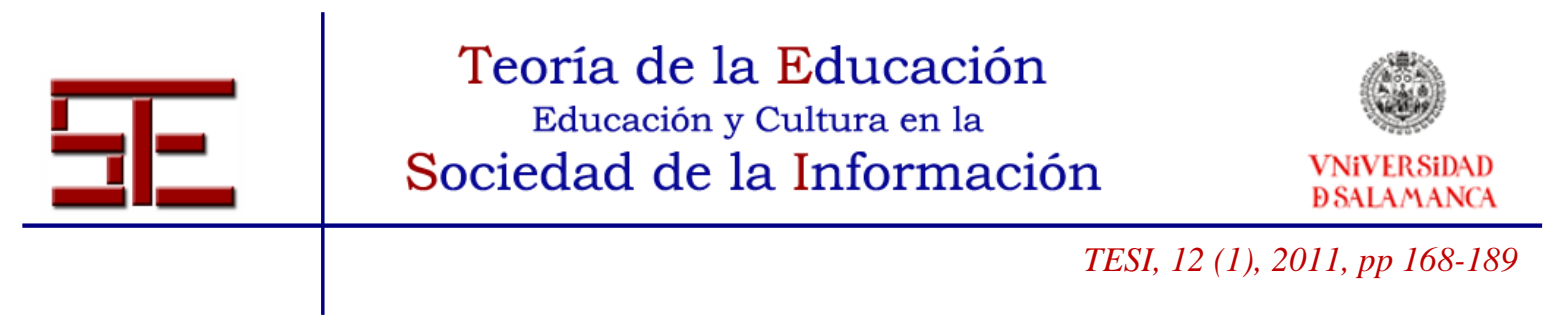

\title{
OPTIMIZATION OF THE INFORMATION BEHAVIOUR BY USING BOOKMARKING TOOLS
}

\begin{abstract}
The current knowledge society and the new education policies in the EU require a new approach to educational resources. The use of a closed and monolithic form such as the textbook has to be replaced by the creation and continuous updating of repositories of resources that facilitate and are part of the learning process. This paper presents an approach to this new paradigm through an analysis of three elements: the research of the information behavior of students in the use of educational resources available on the Net, the functional and usability analysis of the tools that support management and use of information resources, and the pilot of a bookmarking tool in a virtual classroom course on Human-Computer Interaction as practice to improve students' information behavior. Considered together these three elements is key to improving students' information behavior and therefore allow better acquisition of information competencies essential in the current context.:
\end{abstract}

Keywords: learning tools; educational resources tagging; bookmarking; user experience; information competencies.

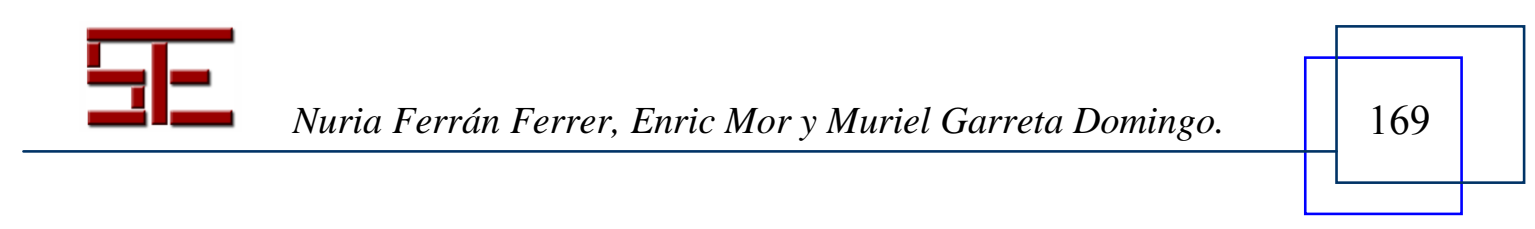




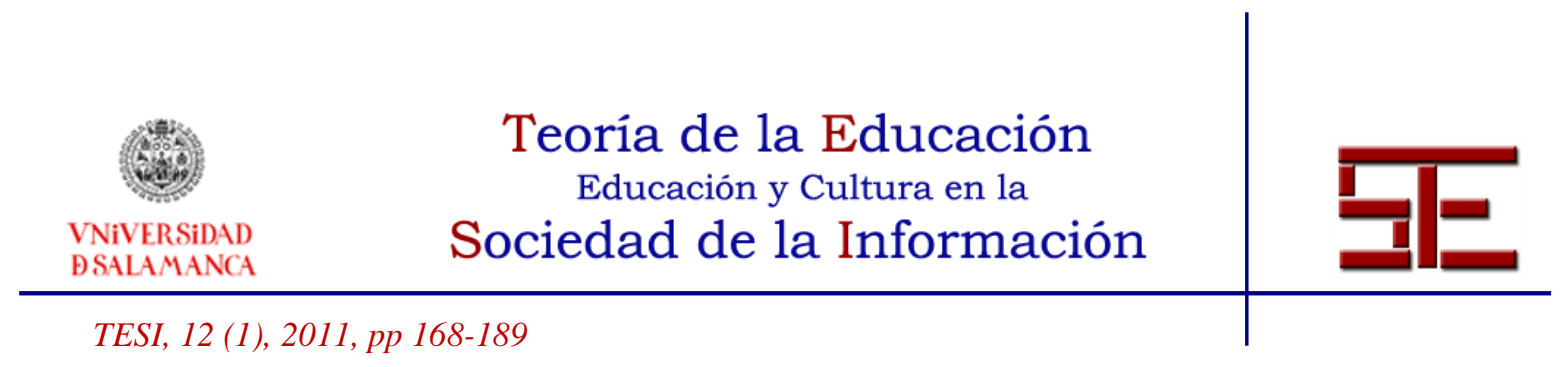

\title{
OPTIMIZACIÓN DEL COMPORTAMIENTO INFORMACIONAL MEDIANTE EL USO DE HERRAMIENTAS DE BOOKMARKING
}

\author{
Fecha de recepción: 18/11/2010; fecha de aceptación: 07/02/2011; fecha de publicación: 31/03/2011
}

\author{
Núria Ferran Ferrer \\ nferranf@uoc.edu \\ Universitat Oberta de Catalunya \\ Enric Mor \\ emor@uoc.edu \\ Universitat Oberta de Catalunya \\ Muriel Garreta Domingo \\ murielgd@uoc.edu \\ Universitat Oberta de Catalunya
}

\section{1.- INTRODUCCIÓN}

Los retos del nuevo Espacio de Educación Superior Europeo y su objetivo de acercar el aprendizaje en la sociedad del conocimiento, el profesorado debe cambiar el rol de dispensador de conocimiento a facilitador de la generación colectiva de aprendizaje y conocimiento. Este cambio de rol supone la transición del modelo educativo y provoca que también cambien los canales por los que se transmitía conocimiento (libros de texto, artículos, etc.).

Este cambio de paradigma conlleva la utilización directa de recursos accesibles y distribuidos en el mundo real y muchas veces disponibles en Internet. De esta manera se pretende alentar a los estudiantes a seleccionar recursos directamente de la Red, utilizarlos, reutilizarlos y compartirlos con el resto de la comunidad educativa. Ante este nuevo modelo educativo en el que el intercambio de contenidos es constante se detectan una serie de desafíos a completar.

Por otro lado, en aquellas disciplinas que requieren una actualización constante de contenidos, especialmente las relacionadas con las tecnologías de la información y la comunicación, es desaconsejable usar un formato de contenidos cerrado, como puede ser un libro, puesto que en un periodo muy breve de tiempo puede verse desfasado. En

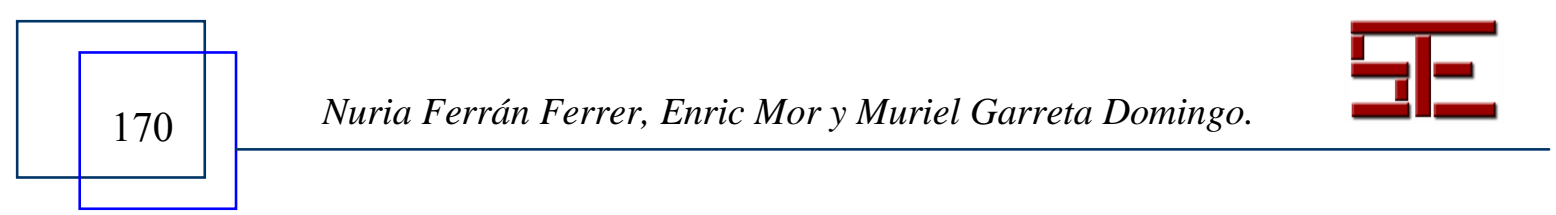




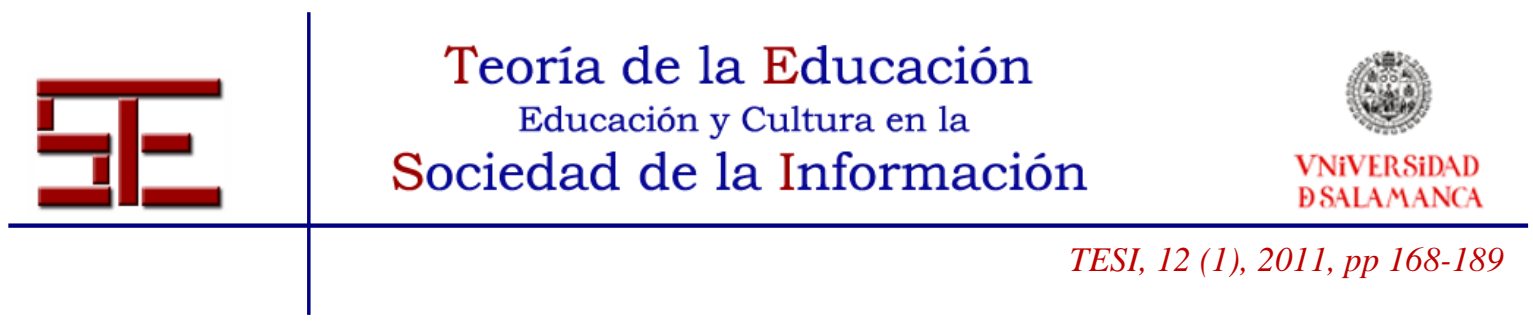

estas asignaturas, estudiantes y profesores deben compartir recursos educativos digitales para mejorar la actualización del aprendizaje. Estos recursos son principalmente contenidos, actividades, artículos y casos prácticos en soporte digital.

En estas asignaturas se llega a producir una construcción social del conocimiento gracias al intercambio de contenidos y recursos y su posterior discusión. Estudiantes y docentes comparten recursos, los comentan, los recomiendan y, en ocasiones, los clasifican y almacenan para su posterior uso y consulta. Estas acciones, especialmente su clasificación, almacenamiento y posterior acceso y consulta, muchas veces constituyen una dificultad añadida y también una dedicación extra de tiempo que debería dedicarse al proceso de aprendizaje. Es el docente quien normalmente realiza el trabajo de clasificar, almacenar y mantener estos recursos y, cuando el docente cambia, muchas veces se pierde este conocimiento acumulado.

Es importante, por tanto, poder disponer de herramientas que faciliten la compartición y construcción social del conocimiento, diseñadas para ser utilizadas en un contexto formativo y que, además, permita su almacenamiento, mantenimiento y futuro uso. Al mismo tiempo es importante que estudiantes y docentes desarrollen un conjunto de competencias digitales e informacionales que les permitan manejarse en esta nueva realidad y hacer frente a los retos que conlleva.

Las llamadas herramientas de marcadores o bookmarking son aplicaciones adecuadas para referenciar y compartir conocimiento en la Red. Estas herramientas se caracterizan principalmente por:

- Guardar y recuperar los enlaces a recursos en la Red.

- Permiten etiquetar y comentar los recursos.

- Valoración o votación de los recursos.

- Normalmente hay un servicio de almacenamiento asociado y toda la información queda almacenada remotamente "en la nube" y, de este modo, un usuario puede acceder a la misma información, en cualquier momento, desde diferentes ordenadores o dispositivos $\mathrm{y}$, además, la información queda a salvo de un posible mal funcionamiento del ordenador personal.

Las herramientas de bookmarking pueden proporcionar, además, una capa de funcionalidades sociales para, de este modo, favorecer la interacción y el intercambio de recursos y conocimiento con otras personas. Esta capa social es muy útil en diferentes aspectos.

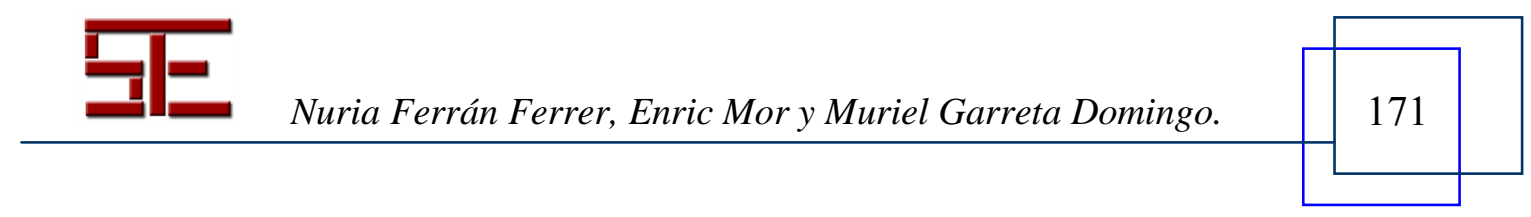




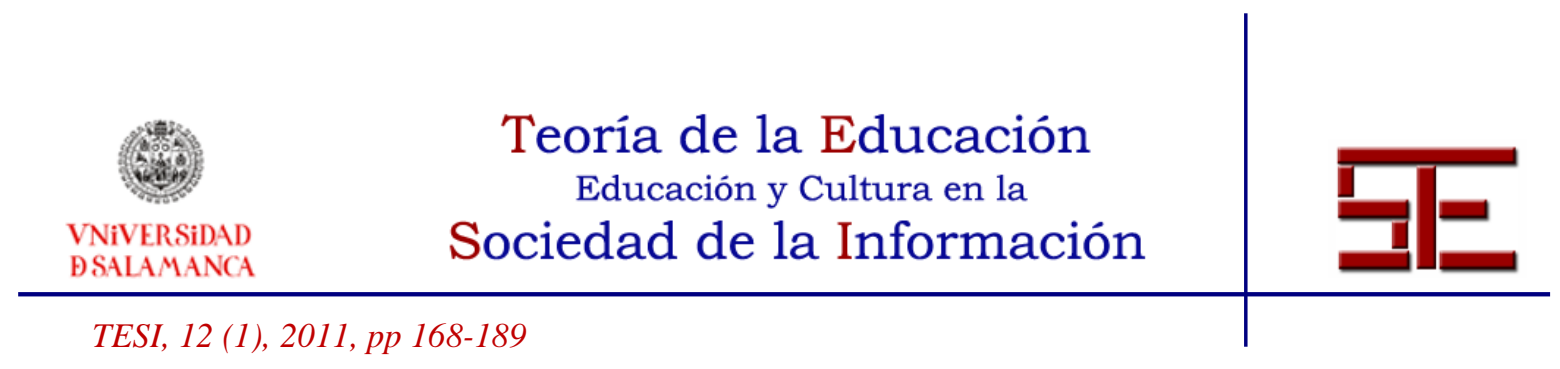

- Compartición de recursos con personas que tienen necesidades e intereses afines.

- Conocimiento de la "popularidad" de un recurso.

- Construcción de folksonomías (clasificaciones colaborativas por medio de etiquetas simples, sin jerarquías ni relaciones entre los términos).

El uso de las funcionalidades que presentan estas herramientas constituye un elemento fundamental en el cambio de paradigma comentado y presentan un conjunto de ventajas e inconvenientes tanto de modo intrínseco por su diseño y usabilidad como en relación a las competencias informacionales de las personas que las han de utilizar. A continuación se presentan con mayor detalle estos aspectos. En la sección 2 se exponen las competencias informacionales necesarias en la sociedad de la información y la sección 3 muestra la situación actual y necesidades a cubrir. En la sección 4 se introduce el estudio y evaluación de las herramientas de bookmarking. En la sección 5 se describe la experiencia educativa llevada a cabo en un aula y, finalmente, en la sección 5 se presentan las conclusiones del presente trabajo.

\section{2.- COMPETENCIAS INFORMACIONALES}

La sociedad actual, llamada sociedad de la información o del conocimiento, se distingue por que es una sociedad en red en que el modo de desarrollo está viviendo un rápido proceso de cambio. La novedad se centra en que la generación de la información, su proceso y transmisión se han convertido en las fuentes de productividad y poder fundamentales de esta sociedad (Castells, 1996).

La educación formal y la formación a lo largo de la vida (lifelong learning) se dan cuenta de estos cambios sociales y económicos del entorno y se encuentran con la demanda de equipar a los profesores, estudiantes y trabajadores con las competencias necesarias para la sociedad del conocimiento y su economía.

De hecho, esta es una de las preocupaciones de la Unión Europa des de la Agenda de Lisboa en que se marcó como objetivo estratégico ser competitivos en la nueva economía estableciendo las nuevas habilidades básicas necesarias para el aprendizaje a lo largo de la vida: habilidades relacionadas con las tecnologías de la información, lenguas extranjeras, cultura tecnológica, emprendería y habilidades sociales (Consejo Europeo, Lisboa, 2000, párrafo 26).

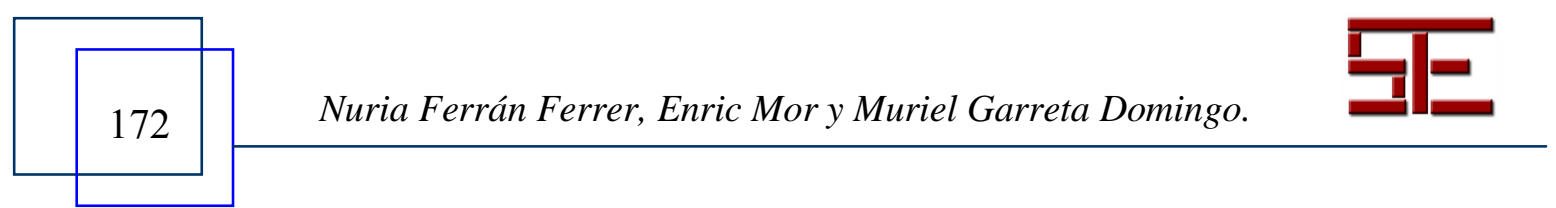




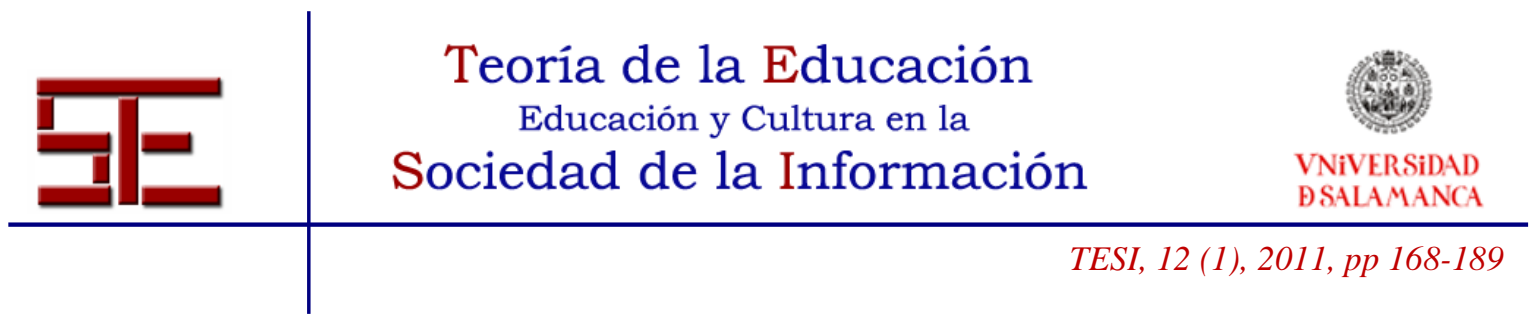

A su vez, para poder "crear" profesionales para la sociedad de la información, el nuevo Espacio Europeo de Educación Superior detectó como indispensable el repensar el aprendizaje y centrarlo en el estudiante, basándose así en la adquisición y desarrollo de competencias en vez de la mera adquisición de contenidos.

Una de las competencias esenciales en la sociedad informacional es la habilidad de encontrar, evaluar, usar y comunicar la información de forma efectiva y eficiente (ALA, 1998). En el ámbito de la Ciencia de la Información estas habilidades se denominan "alfabetización informacional". Pero como hemos visto, en la Agenda de Lisboa lo llamaban "habilidades tecnológicas", también en según qué ámbito se habla de "competencias digitales", "habilidades para el aprendizaje", etc. Así pues, no hay una única definición ni nomenclatura aceptada para definir la alfabetización informacional y hay diversos individuos e instituciones que ofrecen sus definiciones (Ferran ; Virkus, 2010).

A modo de ejemplos, ofreceremos unas definiciones:

Information Literacy encompasses knowledge of one $\mathrm{s}$ information concerns and needs, and the ability to identify, locate, evaluate, organize and effectively create, use and communicate information to address issues or problems at hand; it is a prerequisite for participating effectively in the Information Society, and is part of the basic human right of lifelong learning (UNESCO, 2003).

Information literacy is the adoption of appropriate information behaviour to obtain, through whatever channel or medium, information well fitted to information needs, together with a critical awareness of the importance of wise and ethical use of information in society (Johnston \& Webber, 2003.336).

The concept ,information-related competencies ${ }^{\text {ee }}$ allows to differentiate several blocks of competencies related to information finding, handling and use Information-related competencies defined as the skills, knowledge, attitudes, experience, attributes, and behaviour that an individual needs to find, evaluate and use information effectively (Virkus, 2006).

Breivik considera la alfabetización informacional como un facilitador esencial del objetivo de aprendizaje (2000) y cree que la alfabetización informacional es esencial también para hacer evolucionar el paradigma dominante de información "precocinada" a un nuevo modelo de aprendizaje en que se apodere a los estudiantes con las capacidades para auto-dirigir su aprendizaje a lo largo de la vida. Esta autora junto con Bruce (2002) consideran que los libros de texto, las clases, los ejercicios deberían de

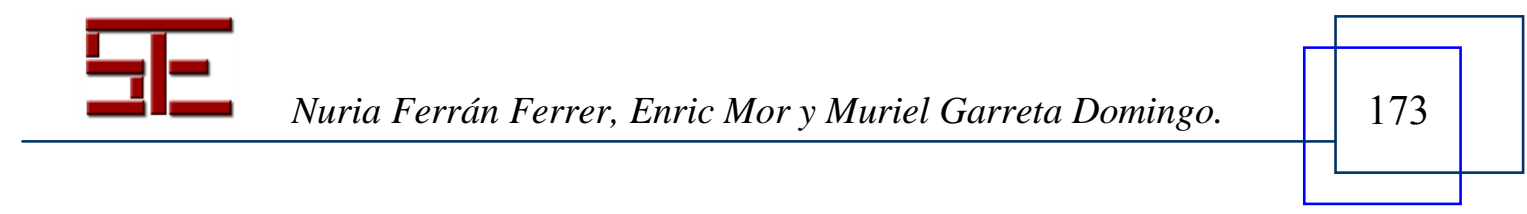




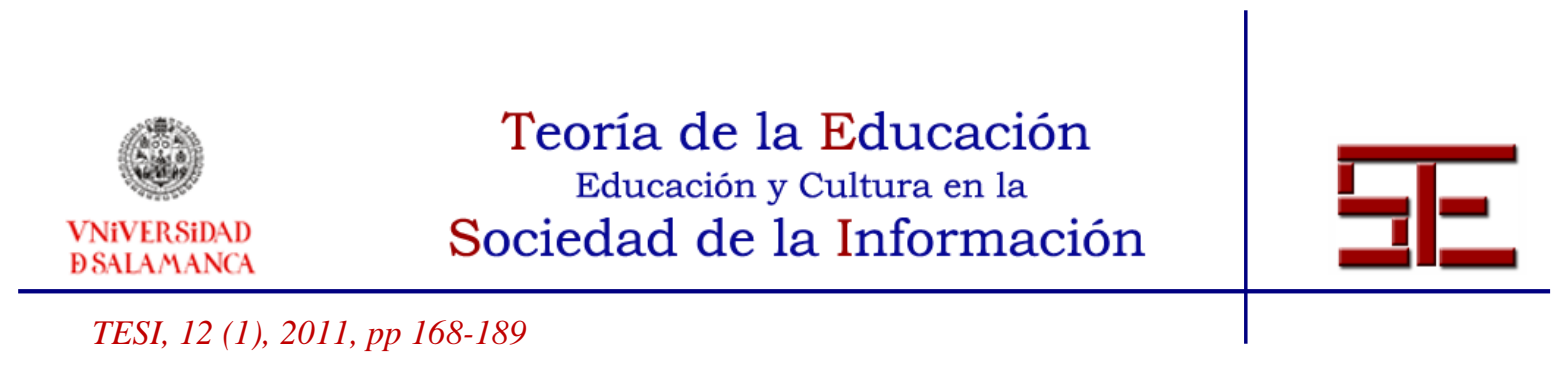

tender a basar el proceso de aprendizaje en los recursos disponibles en la vida real, en los problemas reales del trabajo. No se plantean crear una asignatura específica de formación de las competencias informacionales sino que se plantean reestructurar el proceso de aprendizaje e incluir estas competencias en cada asignatura donde sea necesario el uso de recursos de información, puesto que estas competencias son metodológicas y transversales.

Las competencias informacionales en el ámbito de la educación superior y desde la órbita bibliotecaria se han trabajado mucho y se han llegado a diferentes acuerdos de cuales son las competencias informacionales necesarias para los estudiantes universitarios (ACRL, 2000; SCONUL, 2003).

Las características que deben tener los estudiantes universitarios capacitados informacionalmente contienen tres componentes básicos: el acceso, la evaluación y el uso (Lau, 2006). Por tanto, los individuos con competencias informacionales deben de ser capaces de:

- Determinar la naturaleza de su necesidad informativa.

- Acceder a la necesidad de información de forma efectiva y eficiente.

- Evaluar la información y sus fuentes de forma crítica.

- Incorporar la información seleccionada en su base de conocimiento.

- Usar la información de forma efectiva para conseguir el objetivo propuesto.

- Entender el marco económico, legal y social del uso de la información, su acceso y uso de forma ética y legal (ALA-ACRL, 2004).

SCONUL (2003) añadió dos estándares a estos:

- Clasificar, almacenar y manipular la información recuperada o generada.

- Reconocer la alfabetización informacional como prerrequisito para el aprendizaje a lo largo de la vida.

Y recientemente, hay autores que sugieren que el gran énfasis de las competencias informacionales debe centrarse en el manejo y evaluación de la información en vez de en las competencias relacionadas con el encontrar información (Rowland et al., 2008). Es decir, proponen un giro en la formación tradicional de competencias informacionales.

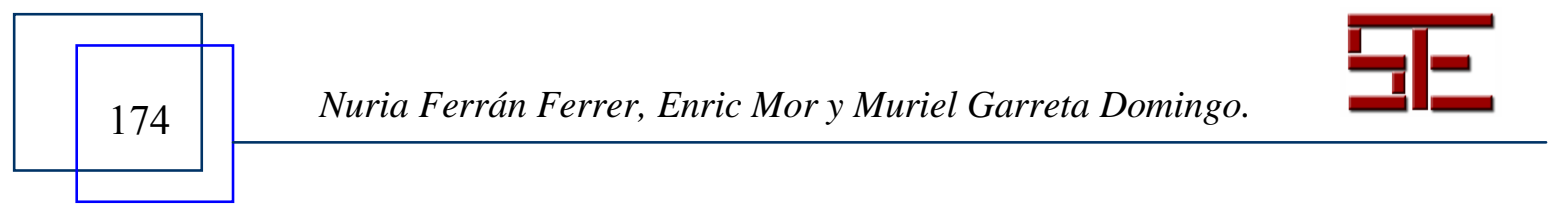




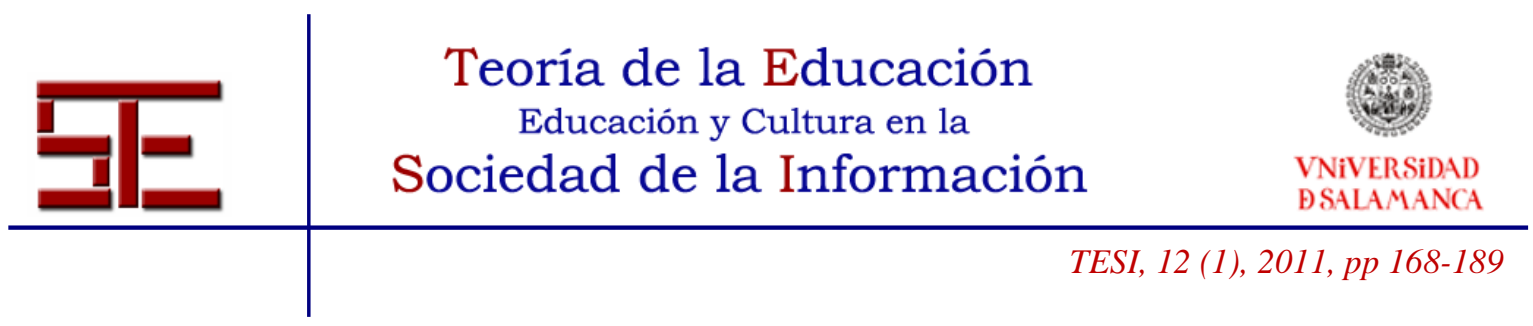

\section{3.- SITUACIÓN ACTUAL Y NECESIDADES}

Cuando hablamos de comportamiento informacional nos referimos a como un individuo ejecuta un conjunto de actividades como son la identificación de necesidades, la búsqueda, la utilización y la transferencia de información (Wilson, 2000). El estudio del comportamiento humano con relación a las fuentes y canales de información y al uso que se hace es de gran interés considerando que estamos inmersos en la sociedad informacional, tal como se ha comentado, en la cual la generación, el procesamiento y la transmisión de la información se convierten en las fuentes fundamentales de la productividad y del poder, debido a las condiciones tecnológicas de este periodo histórico (Castells, 1996). Y desde un punto de vista individual, el estudio del comportamiento informacional es de interés puesto que el análisis de este comportamiento es crucial para desarrollar entornos virtuales (Severance et. al., 2008) que apoyen y promuevan las actividades de acceso, gestión y uso de la información.

En concreto, nuestra investigación pretende estudiar las acciones del comportamiento informacional relacionadas con la gestión personal de la información, es decir las actividades que realiza una persona para adquirir, evitar, crear, almacenar, organizar, mantener, recuperar, utilizar y distribuir la información necesaria para concluir tareas (relacionadas con el trabajo o no) y cumplir diferentes roles y responsabilidades - por ejemplo: ser padre, trabajador o miembro de una comunidad - (Jones, 2007).

El comportamiento informacional se está estudiando de forma intensiva en la disciplina de la Ciencia de la Información desde los años 80 del siglo XX. El aspecto sobre el que se ha estudiado y publicado más del comportamiento informacional es el proceso de búsqueda y recuperación de la información y, en muy menor medida, se han estudiado las acciones personales en relación al uso de la información. Es decir, las actividades que realiza una persona para poder disponer de la información adecuada, en el momento preciso, en el formato justo, con la calidad ideal y la profundidad necesaria para satisfacer una necesidad informativa puntual.

En esta línea, el estudio de las competencias informacionales tiene un sesgo bibliotecario a favor del análisis de las fuentes de información empleadas y las acciones de búsqueda, en detrimento del estudio del comportamiento informacional relacionado con el uso de la información una vez se ha recuperado o se ha recibido.

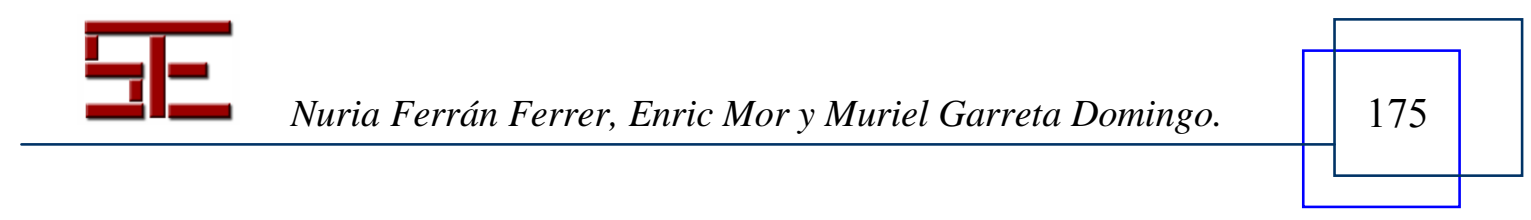




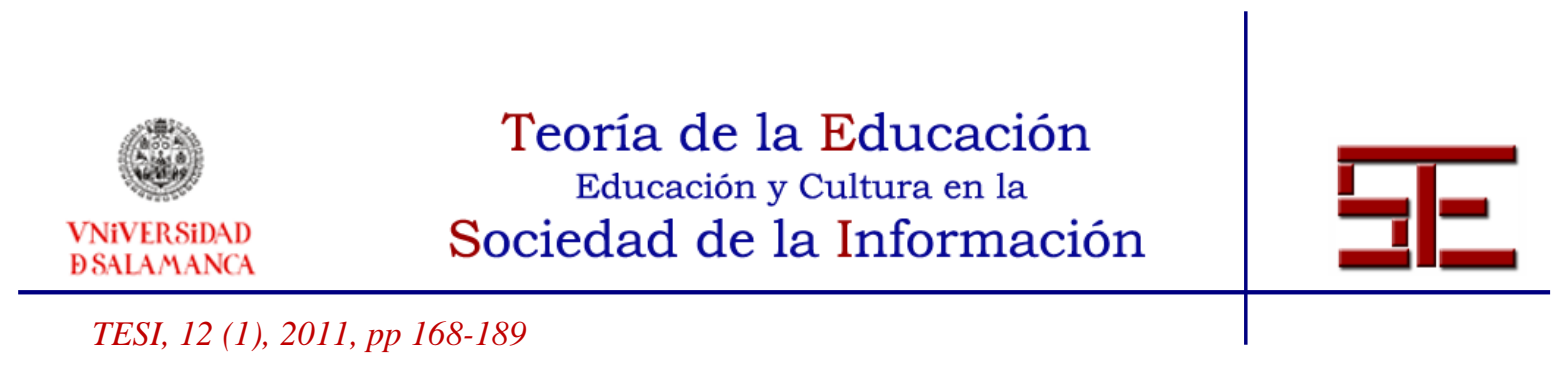

En este contexto, nos planteamos investigar cómo los estudiantes buscan, gestionan y utilizan los recursos de información para entender mejor el comportamiento informacional y cómo diseñar los sistemas de información de manera más usable.

Para obtener el comportamiento informacional de los estudiantes en relación a la reutilización de los recursos localizados por Internet, se diseñó una entrevista estructurada que se mandó a los 65 estudiantes de la asignatura de "Interacción-Persona Ordenador" de la Universidad Oberta de Catalunya (UOC). Primeramente debían realizar una práctica de localización y evaluación de recursos. Posteriormente, mediante la entrevista, se provocaba la reflexión acerca de su manera de gestionar y utilizar los recursos recuperados y cuál sería la manera ideal desde su punto de vista. Las entrevistas fueron la base de un análisis de contenido apareciendo dos comportamientos diferenciados: "comportamiento informacional just-in-time" y "comportamiento informacional just-in-case".

\section{1.- Comportamiento informacional Just-in-case}

Una minoría utilizaban herramientas de bookmarking, la gran mayoría utiliza herramientas de procesamiento de texto y almacena documentos en dispositivos de almacenamiento portátiles o en documentos de GoogleDocs donde copian y pegan en un documento las direcciones o URL de los recursos para recuperarlas posteriormente o se auto-envían las direcciones por correo electrónico para consultarlas cuando tengan más tiempo. Algunos utilizan la opción Favoritos del navegador y otros una libreta donde anotan las direcciones. También el Historial del navegador un recurso utilizado para recurrir a los recursos encontrados en el pasado.

Las herramientas de bookmarking no son ampliamente utilizadas, algunos de los motivos son porque las interfaces son en inglés, que no hay tutoriales de uso. Sólo utilizan herramientas como Delicious.com o Mr. Wong estudiantes que han participado en cursos de formación de herramientas de la llamada Web 2.0 o estudiantes que además de utilizar el bookmarking ya son usuarios activos de RSS, opciones avanzadas de Google o de Netvibes.

Algún estudiante, de edad más avanzada, prefiere imprimir las páginas que considera que son interesantes. O también imprimen los recursos encontrados cuando el uso es para el ámbito personal, sobre todo en la preparación de viajes.

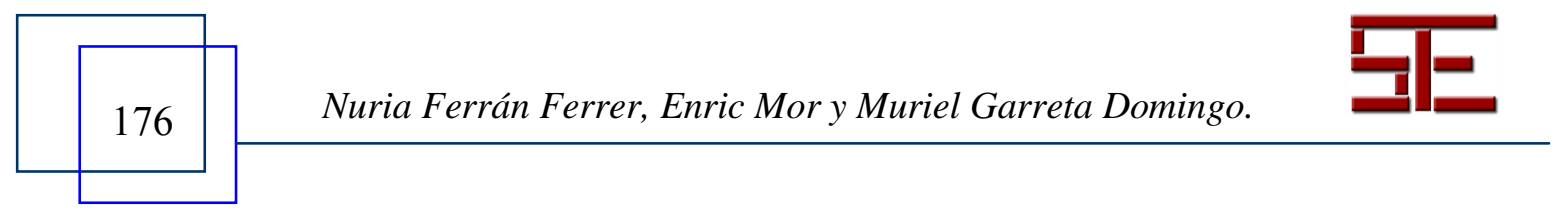




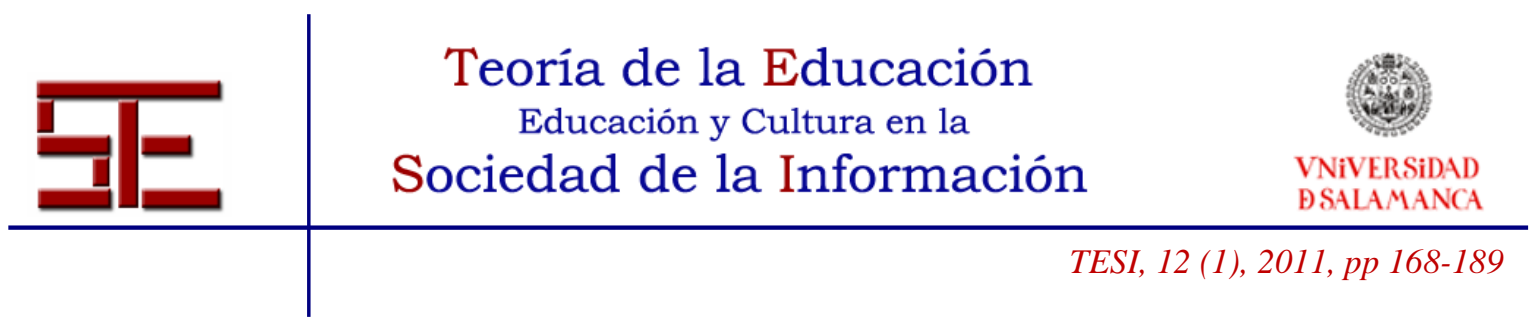

\section{2.- Comportamiento informacional Just-in-time}

Una minoría de estudiantes opinan que es mejor volver a realizar la búsqueda en Internet en vez de invertir tiempo en guardar y clasificar los recursos. Tienen miedo a acumular información. Es como si considerasen Internet como la base de datos y Google como la herramienta de búsqueda.

Hace unos años "Just-in-time" era el paradigma de la nueva gestión de la información, confrontado a su rival "Just-in-case". "Just in case" representa el concepto de información "por si acaso". Una forma de gestión de la información tradicional en que se quiere poseer físicamente los documentos desde una perspectiva que considera que el volumen de información es importante (more is always better).

En cambio el sistema "Just-in-time" representa a la información en un momento dado o en el acto. Es un nuevo paradigma de la gestión de la información que se centra en disponer de la información que se necesita en el momento en que se necesita. No se focaliza en el documento físico ni completo, sino en una modularidad de la información y de granularidad pequeña.

Tanto para el comportamiento que quiere evitar la acumulación de información como el que tiende a acaparar información se presenta como un elemento imprescindible el poder disponer de herramientas que ayuden a localizar, evaluar, almacenar, recuperar, compartir, comunicar, etc., recursos de información electrónica disponibles en Internet.

\section{4.- HERRAMIENTAS DE BOOKMARKING}

La tendencia actual en la que cada vez más los contenidos y materiales educativos se encuentran en soporte digital y distribuidos por la Red conlleva la necesidad de disponer de herramientas con las que los usuarios, los estudiantes y profesores puedan gestionar estos recursos electrónicos. La gestión de estos recursos se basa principalmente en guardar la referencia o la dirección web del recurso y, por otra parte, la recuperación y consulta de los recursos almacenados previamente.

Tal como se ha visto al estudiar las necesidades y competencias informacionales, tanto docentes como estudiantes ponen de manifiesto la necesidad de gestionar recursos y disponer de herramientas para gestionar recursos de información. En la actualidad aunque hay un auge del uso de herramientas de la llamada Web 2.0 en educación (Alexander, 2006), las herramientas de bookmarking apenas se están utilizando en

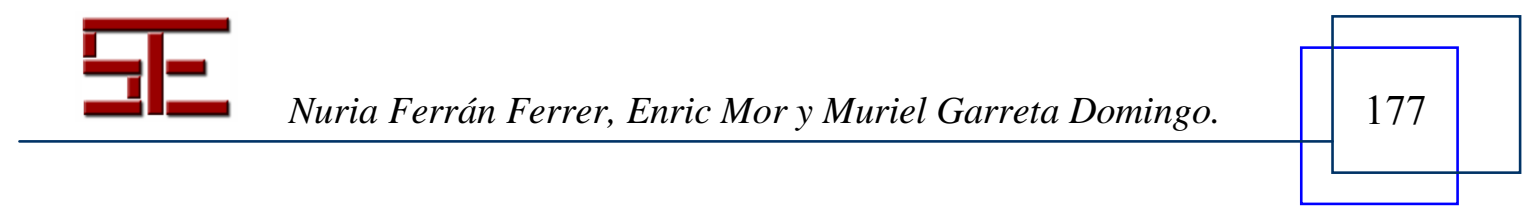




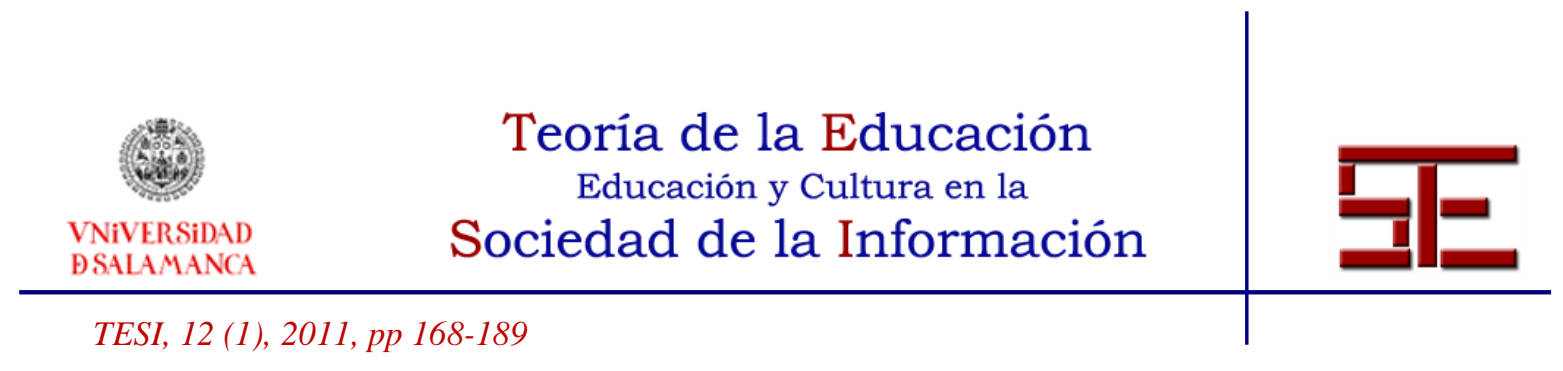

contextos educativos y, consecuentemente, difícilmente se puede encontrar bibliografía que documente experiencias en el aula (Freedman, 2010).

De este modo, con el objetivo de llevar a cabo en el aula una experiencia con el uso de herramientas de bookmarking, se ha realizado una evaluación de las herramientas actuales. Mediante esta evaluación se pretende detectar sus fortalezas y debilidades, tanto a nivel de facilidad de uso como de utilidad, y de este modo diagnosticar aquellas herramientas más adecuadas y su uso idóneo en un contexto educativo. Como resultado de la evaluación se dispondrá de la información necesaria para elegir una herramienta o bien para desarrollar una nueva herramienta que disponga de las funcionalidades adecuadas y proporcione una experiencia satisfactoria.

\section{1.- . Metodología de evaluación}

La evaluación de herramientas que se presenta en este trabajo se ha llevado a cabo utilizando una evaluación heurística, que es un método de evaluación de la usabilidad. Para la elección de este tipo de evaluación se ha tenido en cuenta que la evaluación heurística constituye un método fiable que permite descubrir problemas de usabilidad y proporciona información acerca de las carencias y las fortalezas de las herramientas evaluadas. A continuación se presentan los aspectos principales de la metodología utilizada: la evaluación heurística y los principios utilizados, los expertos que han llevado a cabo la evaluación, la selección de herramientas a evaluar y los indicadores o subheurísticos utilizados en la evaluación.

La evaluación heurística es un método de evaluación de la usabilidad que se usa para identificar problemas de usabilidad en una interfaz (Nielsen, 1990). Este tipo de evaluación se lleva a cabo normalmente por uno o más expertos. Los evaluadores estudian la interfaz y la confrontan con un conjunto de heurísticos o reglas previamente establecidas. Este conjunto de heurísticos se basa en un grupo de reglas ampliamente aceptadas para evaluar aspectos de la usabilidad y la experiencia de usuario de las interfaces.

Los principios heurísticos se estructuran normalmente en forma de listas de comprobación formadas por indicadores para, de este modo, facilitar la práctica de la evaluación. Para cada heurístico, los indicadores se formulan como preguntas, donde la respuesta afirmativa indica la ausencia de un problema de usabilidad.

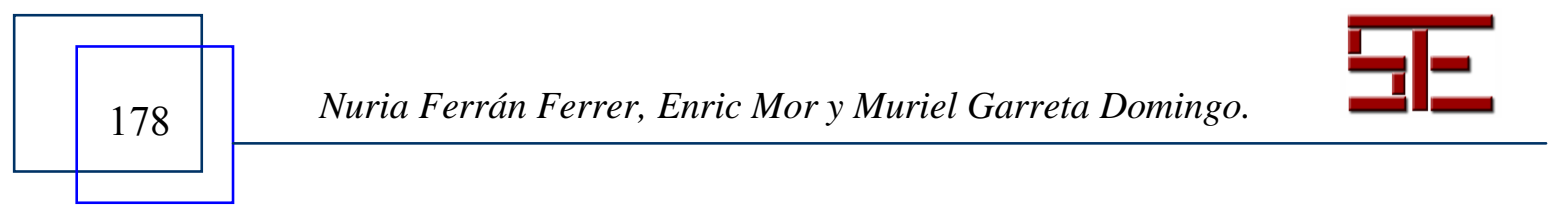




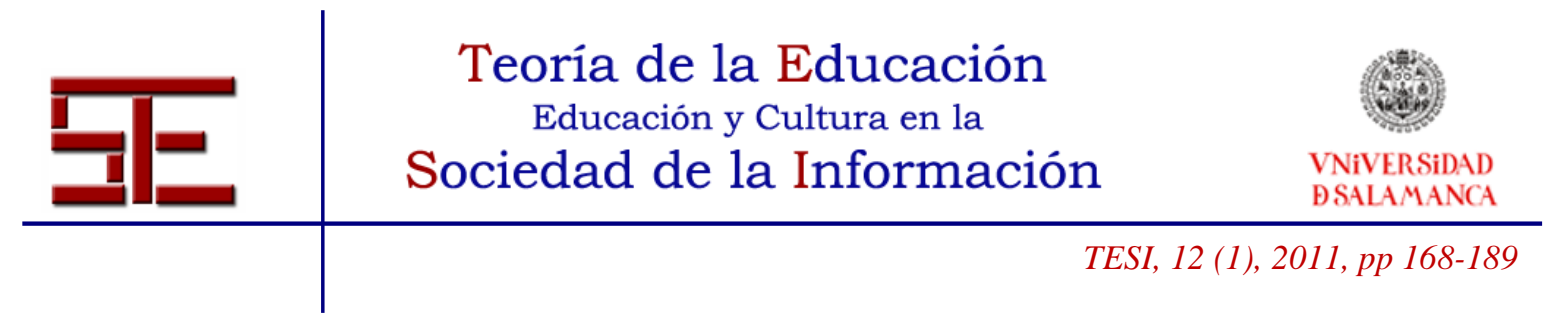

Para evaluar las herramientas de bookmarking se ha utilizado el conjunto de heurísticos de Nielsen puesto que cubren los aspectos principales a evaluar y proporcionan suficiente flexibilidad para adecuar los indicadores a las características de las herramientas en contextos educativos. Las reglas heurísticas propuestas por Nielsen se resumen así (Nielsen, 1993):

1. Visibilidad del estado del sistema (mensajes, feedback).

2. Adecuación entre el sistema y el mundo real (tipo de lenguaje).

3. Libertad y control por parte del usuario (navegación).

4. Consistencia y estándares (gráficos, colores, etc.).

5. Prevención de errores (mensajes, prevención, etc.).

6. Reconocimiento antes que recuerdo (iconos, metáforas, etc.).

7. Flexibilidad y eficiencia en el uso (adaptación a los navegadores, buscador, etc.).

8. Diseño estético y minimalista (sobrecarga, minimalismo, etc.).

9. Ayuda a los usuarios a reconocer, diagnosticar y recuperarse de los errores.

10. Ayuda y documentación (información de apoyo).

En el estudio de usabilidad que se ha llevado a cabo han participado tres evaluadores expertos. Cada experto ha evaluado el conjunto de herramientas seleccionado y las ha valorado en base a los heurísticos y los indicadores definidos para cada uno de ellos. Los resultados que se presentan incluyen el resultado ponderado de la evaluación de cada experto.

\section{2.- Herramientas de bookmarking}

En la actualidad existen numerosas herramientas de bookmarking, casi todas ellas gratuitas y con funcionamiento mediante una interfaz web. Algunas de las herramientasmás interesantes son: Delicious ${ }^{7}$, Blogmarks $^{8}$, Blinklist ${ }^{9}$, Mister-Wong ${ }^{10}$, Evernote $^{11}$, Furl ${ }^{12}$, Diigo ${ }^{13}$ y Google Bookmarks ${ }^{14}$.

www.delicious.com.
www.blogmarks.net.
www.blinklist.com.
www.mister-wong.com.
www.evernote.com.
www.furl.net.
www.diigo.com.

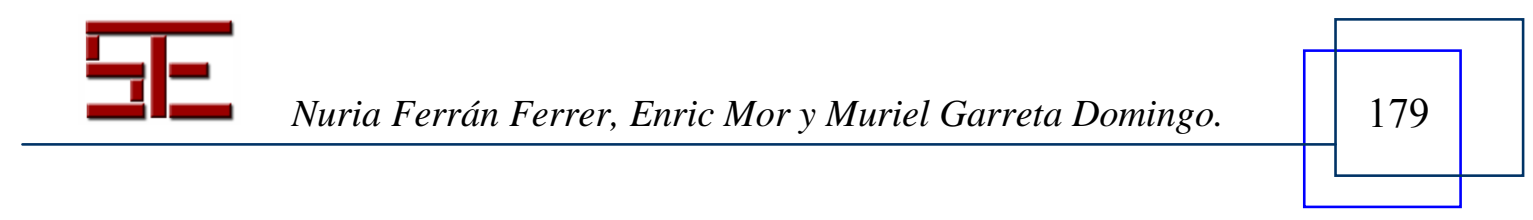




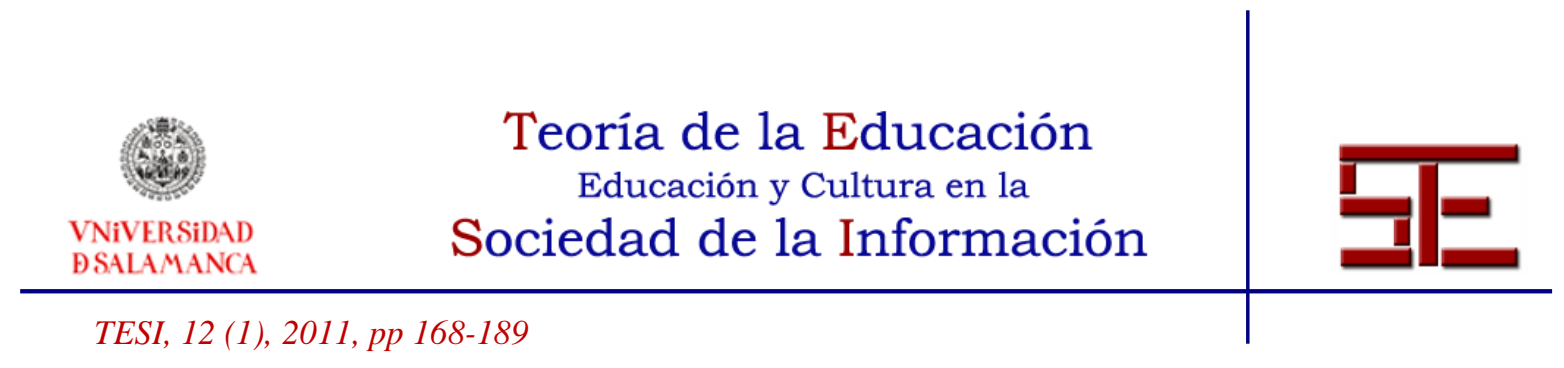

También es interesante resaltar que hay herramientas que originalmente se podrían clasificar como gestores de referencias bibliográficas, y que en la actualidad incorporan también la gestión de enlaces. Algunas de estas herramientas son: Citeulike ${ }^{15}$, Connotea $^{16}$, Bibsonomy ${ }^{17}$ y Zotero ${ }^{18}$.

Para llevar a cabo la evaluación, se han revisado todas las herramientas y se ha seleccionado el subconjunto más representativo a nivel de penetración y de uso. En la selección de herramientas también se han tenido en cuenta las herramientas utilizadas por estudiantes de asignaturas de Interacción Persona-Ordenador de titulaciones en Documentación y en Ingeniería Informática de la UOC. De este modo, para este trabajo se han evaluado tres herramientas: Delicious, Google Bookmarks y Evernote.

Las tres herramientas seleccionadas proporcionan las opciones básicas de las herramientas de bookmarking y, además, otras funcionalidades que se consideran importantes, como son: almacenaje remoto de la información, compartición de los recursos con otros usuarios, publicación de los enlaces a recursos, la capacidad de la herramienta para importar y exportar los datos almacenados, posibilidad de evaluación de la utilidad o calidad de los recursos de uno mismo y de otros usuarios y facilidad de clasificar los recursos con palabras clave. Gracias a estas funcionalidades, las tres herramientas son adecuadas para contextos educativos en general.

Como conclusiones generales, se puede afirmar que, a pesar de las distintas puntuaciones, las tres herramientas presentan aspectos positivos para la gestión de enlaces a recursos de Internet, destacando especialmente Evernote y Delicious.

Teniendo en cuenta la valoración de los expertos, las herramientas mejor valoradas son Evernote y Delicious. Las dos herramientas presentan características que proporcionan versatilidad para ser usadas para almacenar recursos de aprendizaje. Evernote

15 www.google.com/bookmarks/.

15 www.citeulike.org.

16 www.connotea.org.

17 www.bibsonomy.org.

18 www.zotero.org.

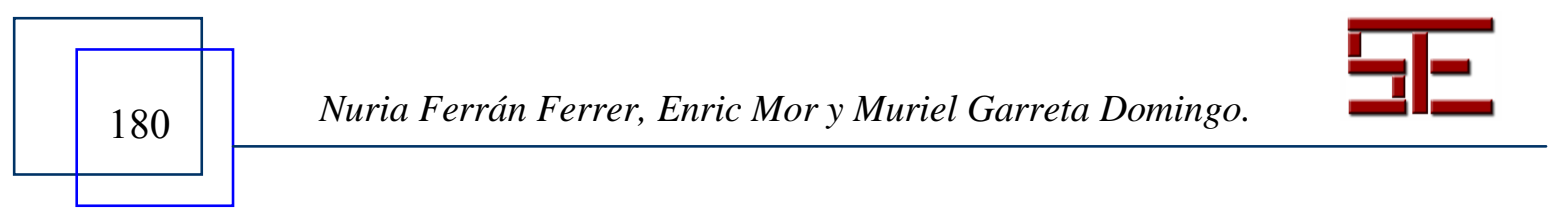




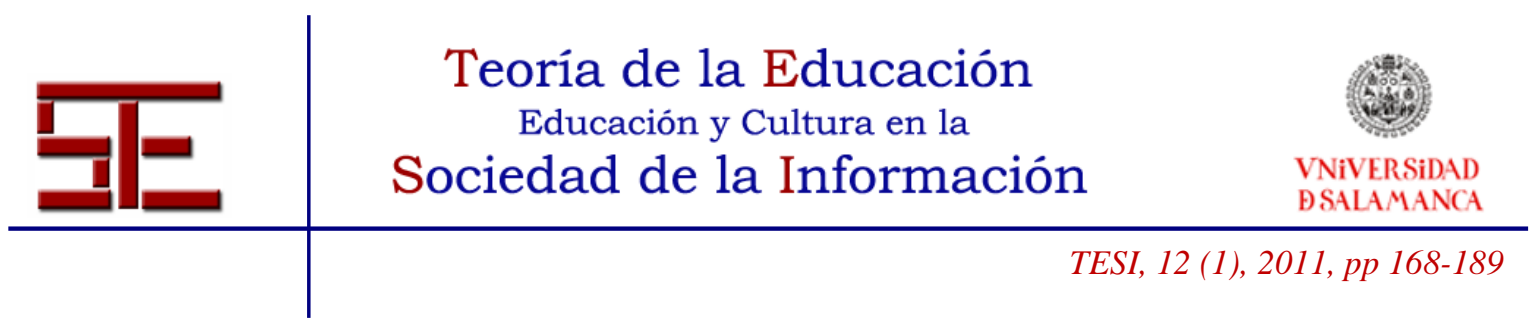

proporciona la funcionalidad adicional de poder almacenar ficheros, tanto de texto como multimedia, y recuperar fácilmente la información de estos ficheros. Esto es un aspecto importante en un entorno educativo puesto que la gestión no solo de la referencia al contenido sino del propio contenido es una competencia informacional necesaria. No obstante este no ha de ser el único aspecto a tener en cuenta a la hora de seleccionar una herramienta. Delicious, por su parte, presenta dos ventajas comparativas importantes: su gran número de usuarios y que permite que se pueda construir una aplicación informática específica para acceder a la información almacenada. Por su parte, el volumen de usuarios de Delicious permite un uso más óptimo de las etiquetas de los recursos y que la herramienta pueda sugerir la etiqueta más adecuada para cada enlace.

De cara a ver el encaje de estas herramientas y su uso en contextos educativos es conveniente recordar que lo importante no es la tecnología sino el uso que las personas, estudiantes y profesores, hacen de ella. Así, es importante ver la flexibilidad que las herramientas ofrecen a los usuarios en relación a la gestión y organización de recursos $\mathrm{y}$, especialmente, en su proceso de aprendizaje (Holmes, 2006).

Un aspecto importante de cara a facilitar el uso de herramientas de Internet a estudiantes y docentes es el método de registro inicial e identificación de los usuarios de la herramienta. Cada estudiante y cada docente deben darse de alta en la herramienta que quieran utilizar, proporcionando sus datos personales y accediendo posteriormente mediante un nombre de usuario y una palabra clave. Si, además, se desean utilizar los aspectos sociales de compartición de información de la herramienta, será necesario que un mismo grupo de estudiantes se ponga de acuerdo para registrarse y utilizar una misma herramienta. Si se dispone de un entorno virtual de aprendizaje, muchas veces la mejor solución será integrar la herramienta de bookmarking en el propio entorno virtual y que estudiantes y docentes puedan acceder a él con el nombre de usuario del que ya disponen; sin necesidad de volver a registrarse.

Otro aspecto a destacar de las herramientas de bookmarking es que aunque las tareas fundamentales a llevar a cabo, guardar, recuperar y buscar enlaces, son aparentemente sencillas, éstas esconden cierta complejidad. Con las herramientas actuales es muy sencillo almacenar información y, al mismo tiempo, puede llegar a ser muy difícil buscar y recuperar la información almacenada. De este modo se puede decir que el diseño actual de las herramientas favorece estrategias y comportamientos informacionales del tipo "just-in-case", es decir, se facilita el almacenamiento masivo de recursos que, en muchas ocasiones, no se volverán a utilizar de nuevo.

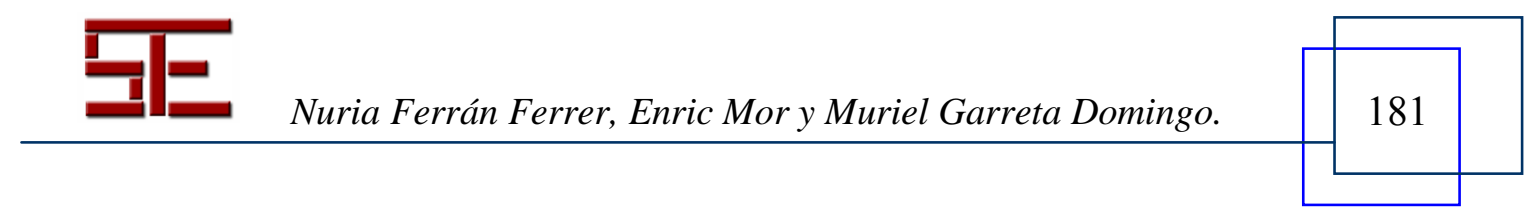




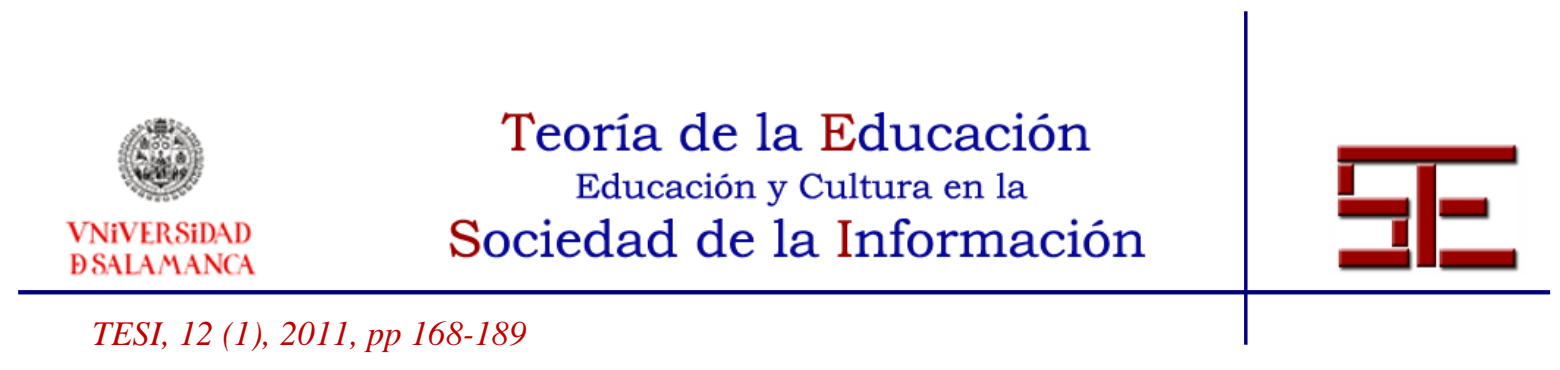

En entornos educativos es recomendable usar herramientas de bookmarking adaptando su interfaz y funcionalidades a cada necesidad específica, que tenga en cuenta el entorno virtual de aprendizaje manteniendo la facilidad de almacenar recursos y favoreciendo y priorizando su recuperación, tanto por búsqueda como por navegación.

La integración de una herramienta de bookmarking en un sistema de e-learning debe tener siempre en cuenta las tres dimensiones que presentan estos sistemas (Casas, 2003): los usuarios, el sistema de gestión e interfaz y el curso o cursos formados a partir de los recursos docentes disponibles. Los usuarios son principalmente los estudiantes y los docentes. El sistema de gestión se apoya en bases de datos y la interfaz permite la interacción de los usuarios con el sistema presentando la información adecuada a cada tipo de usuario. Los cursos están formados principalmente por contenidos y recursos educativos que, al mismo tiempo, están estructurados en objetos de aprendizaje y disponibles en el sistema o distribuidos por Internet.

Utilizar una herramienta de bookmarking, o cualquier otra herramienta, en este contexto implica adecuar la herramienta a los usuarios, integrarla con el sistema gestor y su interfaz y que se adapte a los objetivos y competencias que persigue el curso o programa académico. En consecuencia, no todas las herramientas de bookmarking existentes se pueden integrar y utilizar en un contexto educativo determinado y en estos casos será deseable desarrollar una nueva herramienta con un diseño que se adapte a las necesidades específicas.

\section{5.- PRUEBA PILOTO: INTRODUCCIÓN DE UNA HERRAMIENTA DE BOOKMARKING EN EL AULA VIRTUAL}

A continuación se presenta una experiencia educativa a partir de la prueba piloto consistente en la introducción de una herramienta de bookmarking en un entorno educativo. Esta experiencia se ha llevado a cabo en una asignatura la UOC, institución que presenta la característica particular de ser una universidad a distancia completamente en línea con un entorno virtual de aprendizaje propio y un modelo educativo específico.

La evaluación de herramientas de bookmarking existentes ha permitido constatar que aunque funcionalmente son útiles para ser utilizadas en algunas asignaturas, presentan

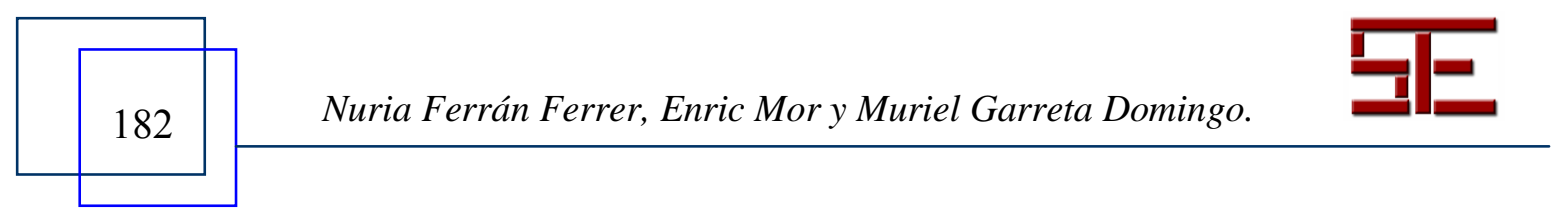




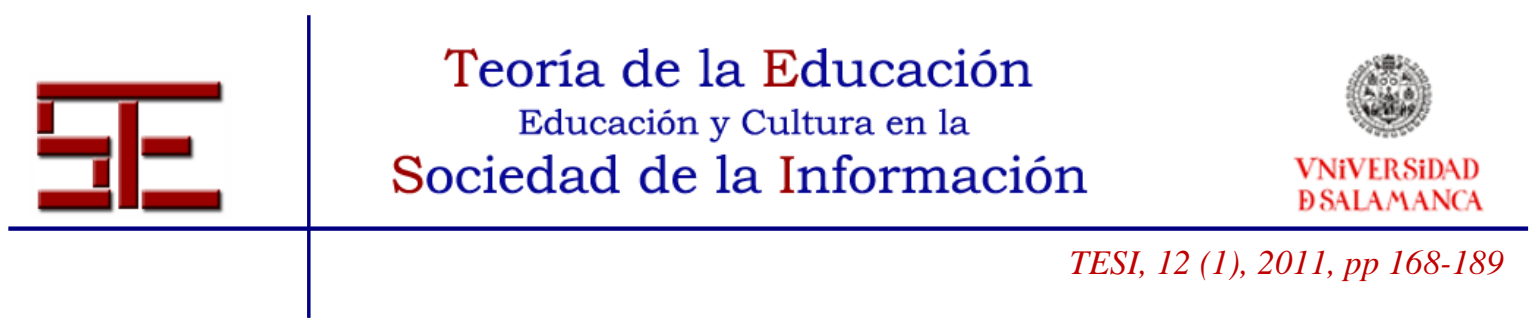

dificultades de integración y adaptación a las características del entorno virtual de aprendizaje de la UOC y las necesidades específicas de los estudiantes. Por este motivo se decide integrar una herramienta de bookmarking (SemanticScuttle) ${ }^{19}$ que tenga en cuenta los aspectos clave de la evaluación de herramientas realizada. Principalmente se persigue disponer de una herramienta que se integre con el entorno virtual de aprendizaje de modo que los usuarios puedan acceder a ella directamente sin necesidad de darse de alta en otra herramienta con otro nombre de usuario y palabra clave. También se requiere una herramienta que aunque presente menos funcionalidades que las disponibles comercialmente, estas sean más claras y fáciles de utilizar en relación al proceso de aprendizaje, especialmente se busca que la herramienta facilite tanto el almacenamiento de recursos como su recuperación al cabo del tiempo.

El objetivo principal de la prueba piloto diseñada es mejorar la calidad del aprendizaje de las asignaturas del área de conocimiento de Interación Persona-Ordenador (IPO), facilitando la adquisición de competencias informacionales y fomentando la formación de una comunidad de creación social del conocimiento mediante una herramienta de compartición de recursos de aprendizaje. Para ello se considera necesaria la integración de una herramienta abierta, colaborativa, centrada en el usuario, que permita a estudiantes y docentes compartir, etiquetar y comentar, de forma ordenada $\mathrm{y}$ contextualizada a un área de conocimiento, los recursos de aprendizaje disponibles tanto en el espacio de aprendizaje como en toda la Red.

Para llevar a cabo el piloto se ha diseñado y se ha puesto en marcha una herramienta de bookmarking de software libre que proporciona las siguientes funcionalidades principales: el acceso a los recursos mediante la herramienta es múltiple: secuencial, buscador y nube de etiquetas. Además, la herramienta permite compartir y visualizar los enlaces añadidos por los usuarios y la votación de los recursos que se consideran más interesantes. Para próximas versiones de la herramienta se espera que también se pueda navegar por una clasificación de contenidos. De esta manera, el objetivo es que la herramienta se pueda adaptar a las necesidades que en cada momento tengan sus usuarios. El equipo docente supervisará el etiquetado y clasificación de recursos reforzando aquellas etiquetas más adecuadas y ajustando la situación del recurso a la taxonomía. Esta herramienta, por tanto, permitirá ordenar la arquitectura conceptual de

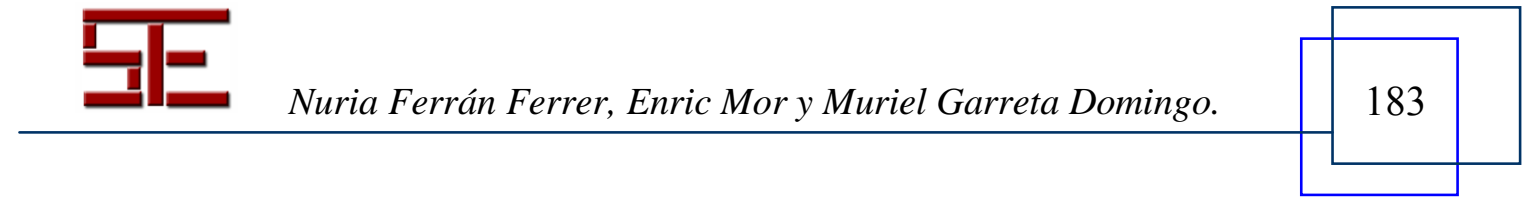




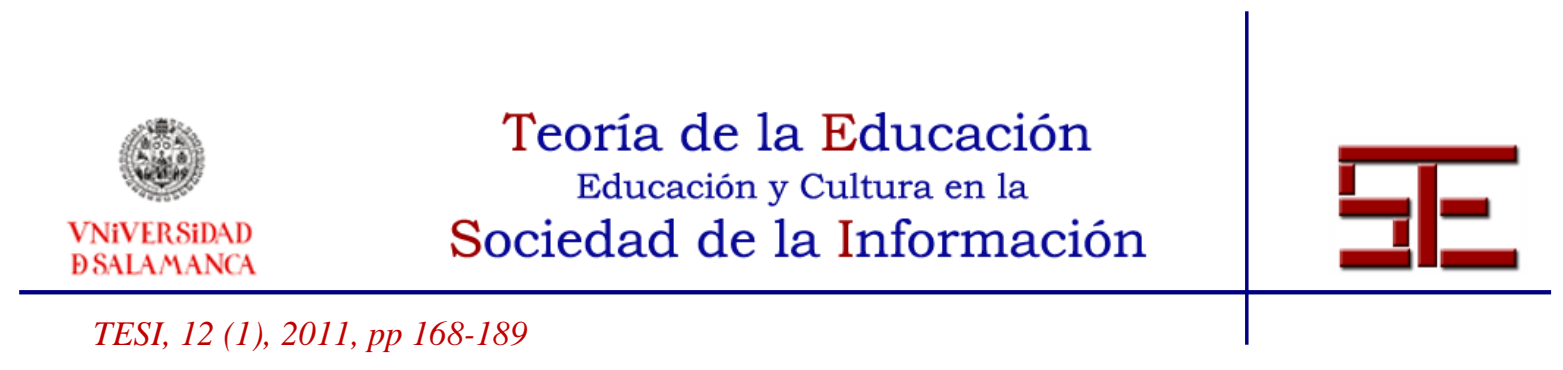

los contenidos de las asignaturas de Interacción Persona-Ordenador, simplificar el proceso de creación y publicación de nuevo material educativo al equipo docente, realizar consultas y localizar, de forma eficiente, los recursos que pueden ser útiles para cada actividad e implicar la participación de toda la comunidad educativa (estudiantes y docentes).

Los estudiantes de la asignatura Interacción Persona-Ordenador han tenido acceso a la herramienta a partir de la segunda mitad del curso académico coincidiendo con una actividad de aprendizaje. Esta actividad consistía en trabajar competencias relacionadas con la accesibilidad. La accesibilidad es una temática muy relacionada con la tecnología y en la web pueden encontrarse informaciones más actualizadas que en los materiales didácticos de la asignatura. Para promover la utilización de la herramienta de bookmarking para gestionar los enlaces a recursos de accesibilidad, se añadió un requisito a la actividad, con repercusión en la nota final. En esta actividad, cada estudiante debía buscar en Internet al menos dos recursos relacionados con tecnología y accesibilidad y añadir los recursos a la herramienta indicando sus palabras clave. Además, se pedía a cada estudiante el valorar recursos ya existentes en la herramienta, utilizando la opción de votar favorable o desfavorablemente un recurso.

En el aula de Interacción Persona-Ordenador donde se ha llevado a cabo la experiencia, hay 65 estudiantes matriculados. 47 estudiantes han participado en la actividad añadiendo 81 enlaces a recursos. El primer dato observable es que no todos los estudiantes han añadido los 2 recursos que pedía en la actividad. Otro aspecto a destacar es que apenas han utilizado palabras clave para etiquetar los recursos añadidos, limitándose a poner una o dos etiquetas y, en la mayoría de los casos, ninguna. En relación a los votos a recursos, 6 de los recursos acumulan el mayor número de votos, mientras que el resto de enlaces tienen uno o ninguno.

\section{6.- CONCLUSIONES Y FUTURA INVESTIGACIÓN}

En este trabajo se ha presentado una experiencia que ha consistido en introducir y evaluar una herramienta que permita a la comunidad de aprendizaje compartir recursos de aprendizaje en formato digital y que estos puedan ser enlazados, comentados, etiquetados y clasificados. Para llevar a cabo esta experiencia se han realizado entrevistas en profundidad para establecer el comportamiento informacional de los estudiantes y se ha elaborado un informe de evaluación de las herramientas de bookmarking más representativas. Finalmente, se ha integrado una herramienta de

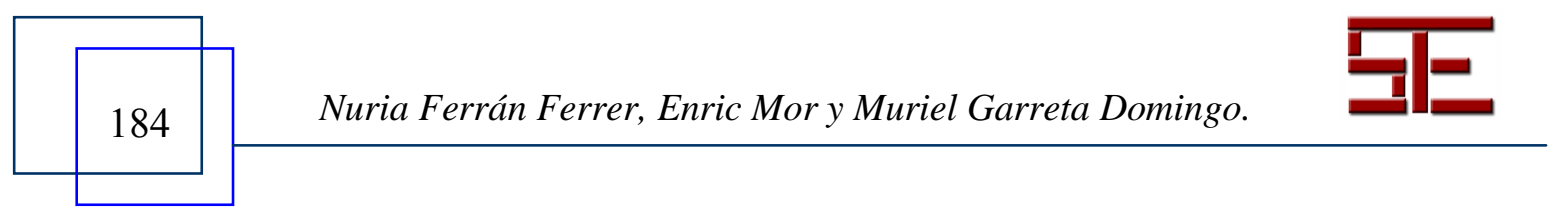




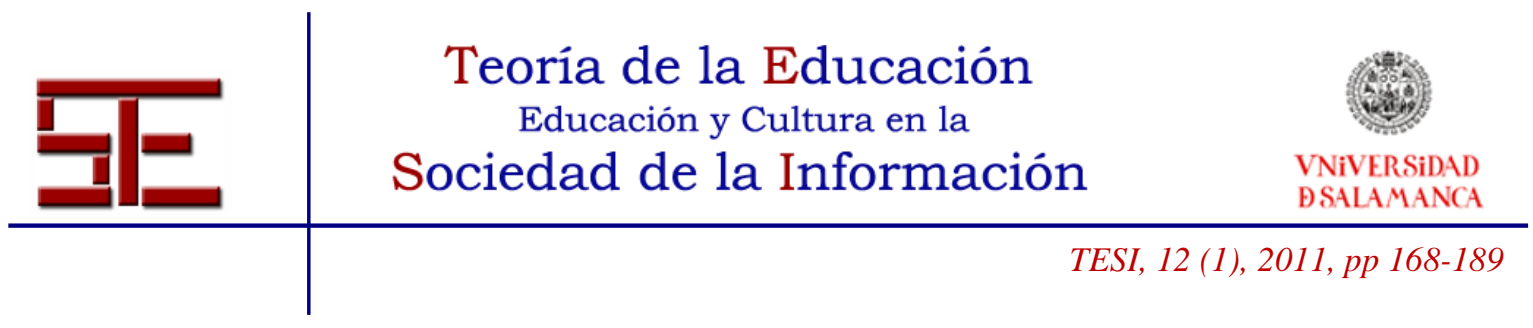

bookmarking en aula virtual y se ha analizado su uso en dicha comunidad de estudiantes.

Los resultados muestran el bajo índice de utilización de herramientas específicas de gestión de recursos de información. Por ello y con el objetivo de promover dichas herramientas así como la adquisición de las competencias informacionales asociadas, el uso de estas herramientas debe ser obligatorio y con consecuencias en la evaluación de los estudiantes. Se deberán establecer todas las acciones a realizar con cada recurso electrónico para asegurar la plena utilización de la herramienta de bookmarking y en consecuencia conseguir una información suficientemente relevante sobre la utilización de este tipo de herramientas.

La utilización total de todas las prestaciones de este tipo de herramientas también permitirá hacer análisis de las folksonomías utilizadas en la clasificación de los recursos. Por un lado, a los docentes les permitirá conocer las relaciones conceptuales que establecen los estudiantes durante el proceso de aprendizaje y a los estudiantes les permitirá seguir un proceso personalizado del aprendizaje evitando la secuencialidad habitual de los contenidos. La implementación de la herramienta en la prueba piloto ha puesto de manifiesto que los estudiantes no han utilizado adecuadamente el etiquetado de recursos. Esto pone de manifiesto problemas de diseño de la herramienta a nivel de navegación por etiquetas puesto que en primer lugar se presentaban las etiquetas personales de cada usuario y quedaba en un segundo plano sin aclaración la presentación de las etiquetas globales, resultando en una confusión en la navegación y el uso de etiquetas.

La facilidad de expresar la opinión sobre la calidad y la utilidad de los recursos electrónicos que ofrecen las herramientas de bookmarking hacía creer, inicialmente, que se obtendría una colección digital de recursos de aprendizaje de calidad, teniendo en cuenta que la selección de los recursos la hacían estudiantes en la materia bajo supervisión docente. Pero la prueba piloto ha puesto de manifiesto que al no haberse entendido la navegación entre los recursos, se han ponderado siempre los mismos enlaces. Para el próximo curso, cuando pueda mejorarse el diseño de navegación de esta herramienta, se considera que la colección digital de recursos sería un contenido muy interesante para dos tipos de usuarios. Por un lado, los estudiantes de la misma asignatura del próximo semestre $\mathrm{y}$, por otro lado, si los contenidos se publican en abierto, cualquier persona interesada en la materia puesto que la calidad de la selección viene contrastada por una comunidad académica. Asimismo, garantizando la formación

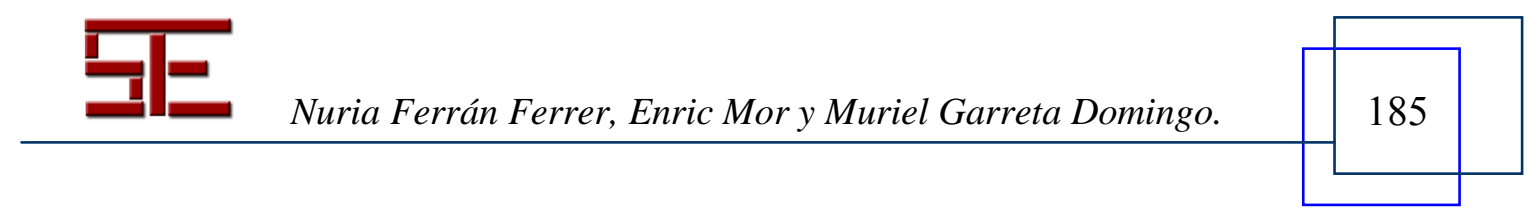




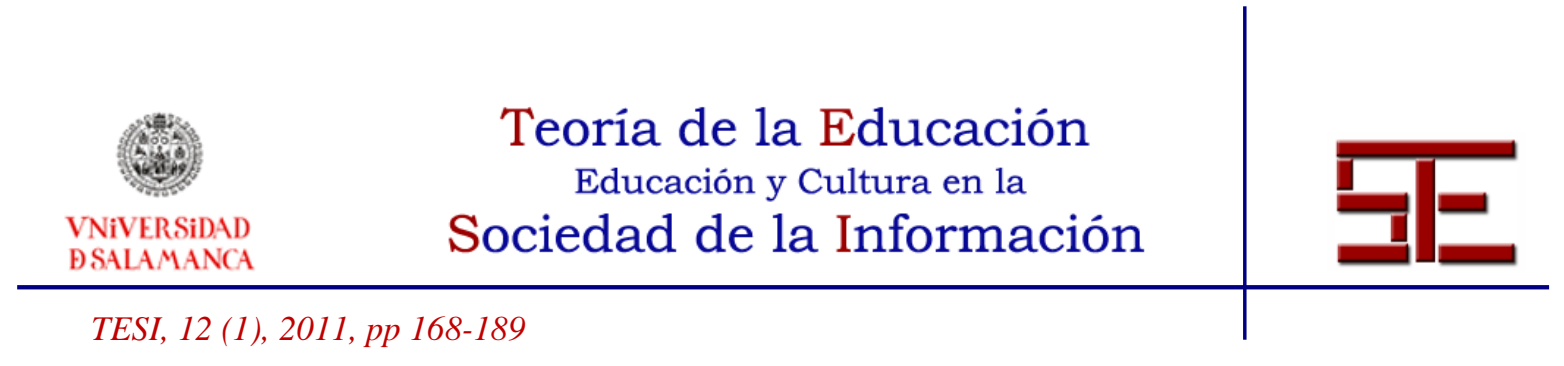

en competencias informacionales a los estudiantes (buscar, seleccionar, evaluar, etiquetar, etc.), también se garantiza una mejor calidad de la colección.

Teniendo en cuenta los dos tipos de comportamiento informacional detectados en este proyecto de investigación, puede interpretarse que las ventajas que ofrecen estas herramientas para la gestión y uso de la información no son consideradas lo suficientemente ventajosas para invertir tiempo en su utilización y, por consiguiente, modificar el comportamiento informacional. Específicamente, el comportamiento "justin-case" no utiliza las herramientas específicas de bookmarking aunque sí utiliza otro tipo de herramientas tecnológicas más simples, más conocidas y habituales, con las que se siente más cómodo. El comportamiento informacional "just-in-time" no utiliza herramientas específicas puesto que no ha encontrado un sistema lo suficientemente rápido, ágil o con prestaciones más interesantes como para sustituir un buscador o navegador de Internet.

La prueba piloto llevada a cabo ha permitido observar elementos importantes a tener en cuenta de cara a mejorar la experiencia. Aunque los estudiantes han tenido a su disposición una herramienta de bookmarking integrada y a distancia de un clic, ésta no ha sido su herramienta de preferencia para gestionar enlaces. Esto es debido, por una parte, a que la herramienta no ha estado disponible desde el inicio de curso y los estudiantes han preferido no cambiar sus estrategias de gestión de información. Únicamente han utilizado la herramienta cuando se han visto obligados por una actividad con repercusión en la calificación final. Por otra parte, la herramienta se ha probado en el marco de una prueba piloto y aunque ofrece las funcionalidades adecuadas, presenta algún problema de diseño, poniendo de manifiesto la importancia de la usabilidad de las herramientas tecnológicas para proporcionar una buena experiencia educativa.

Para una futura integración de herramientas de bookmarking en el aula virtual, hará falta que el profesor especifique la necesidad de etiquetar los contenidos con al menos tres etiquetas, que se dé puntuación a los recursos, etc. Es decir, que se den indicaciones de cómo utilizar al máximo estas herramientas para poder evaluar realmente su introducción. Dichas indicaciones así como la mejora de la usabilidad de la herramienta son aspectos fundamentales para fomentar su utilización y conseguir que su uso se convierta en parte activa del proceso de aprendizaje.

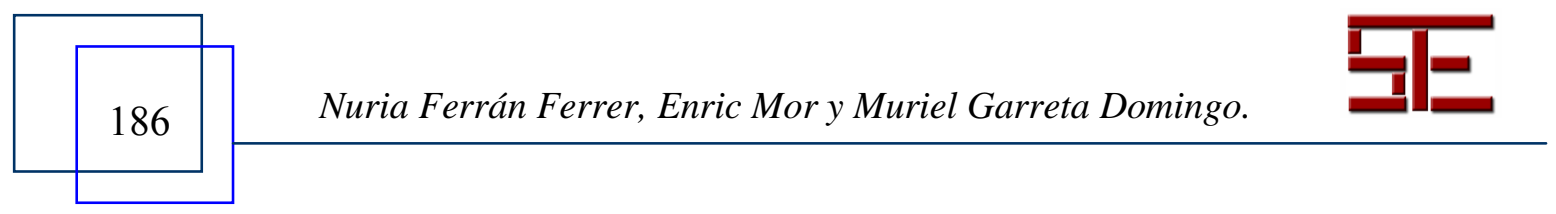




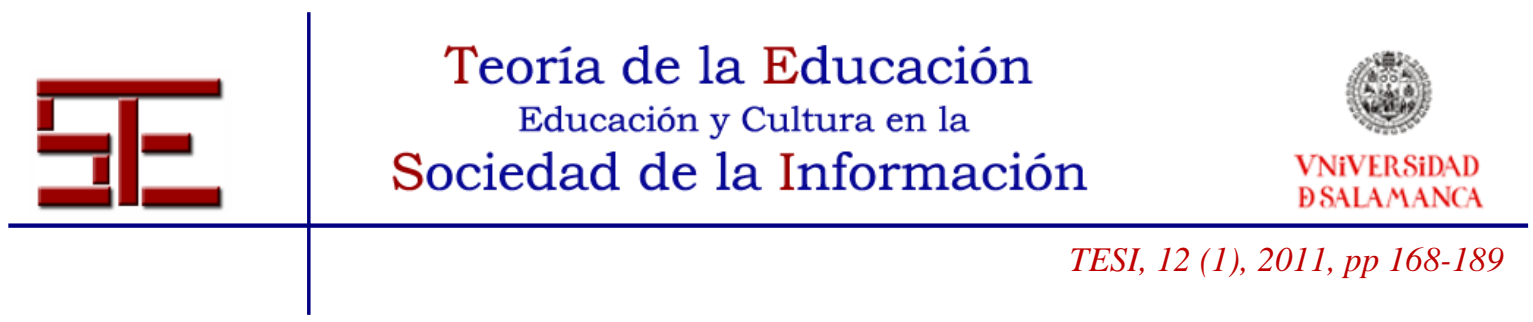

En las próximas líneas de trabajo se continuará explorando la integración de herramientas de bookmarking en los espacios docentes incluyendo el análisis de aplicaciones como Instapaper ${ }^{20}$. Esta aplicación permite guardar páginas web para leer en otro momento. Sin embargo, a diferencia de las herramientas de bookmarking analizadas en este artículo y que podrían caracterizarse como web 2.0, Instapaper no sólo marca como favorita la página sino que también guarda una copia del contenido para permitir su posterior lectura, tanto online como offline. De este modo, vemos como Instapaper se adapta mejor al comportamiento de los usuarios "just-in-case" así como a las necesidades de movilidad de los estudiantes de la UOC y al uso de nuevos dispositivos en el proceso de aprendizaje.

El trabajo futuro a realizar incluye también la elaboración de documentación para estudiantes y docentes con indicaciones pedagógicas y tecnológicas que faciliten el uso de la herramienta y los conceptos de etiquetado, votación y comentario de recursos compartidos. También se esperan establecer los mecanismos de generalización y de difusión que permitan transferir tanto la herramienta como su documentación a otras disciplinas y entornos de aprendizaje.

\section{Agradecimientos}

Este trabajo ha sido parcialmente financiado mediante un Proyecto de Innovación Docente en la convocatoria 2010 de la Oficina Abierta de Innovación de la UOC. Los autores expresan su especial agradecimiento a los estudiantes que participaron en las entrevistas y la prueba piloto de la herramienta de bookmarking.

\section{7.- BIBLIOGRAFÍA}

ALA (American Library Association) (1998). A Progress Report on Information Literacy: An Update on the American Library Association Presidential Committee on Information Literacy: Final Report. Chicago: American Library Association. En http://www.ala.org/ala/acrl/acrlpubs/whitepapers/progressreport.htm.

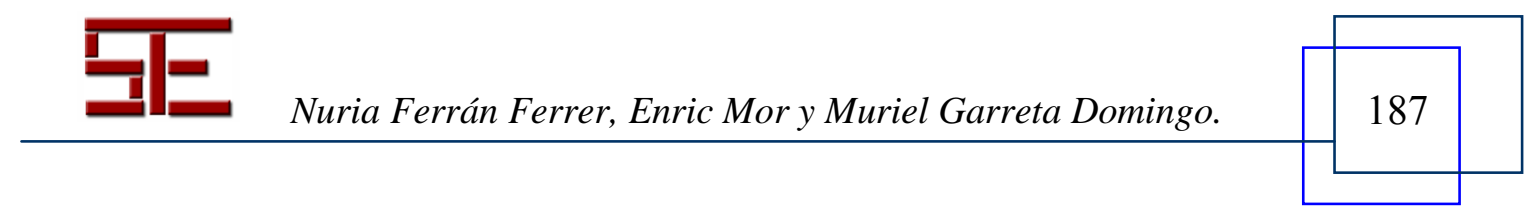




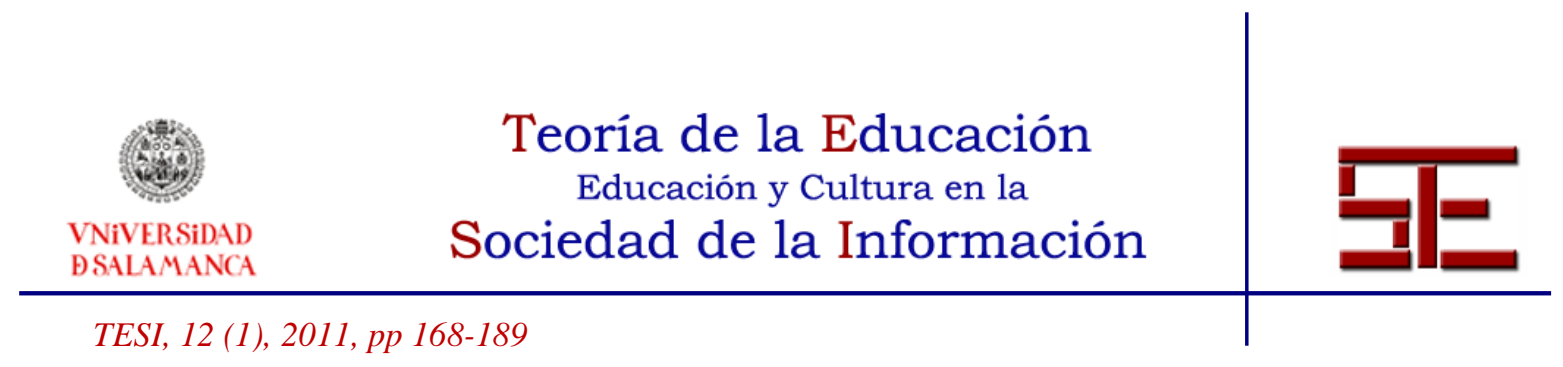

- (2004). Information Literacy Competency Standards For Higher Education. En http://www.ala.org/acrl/

Alexander, B. (2006). Web 2.0: A new wave of innovation for teaching and learning. Learning. 41, 32-44.

Breivik, P. (1998) Student learning in the information age. Arizona: ACE, Oryx Press.

Bruce, C. S. (2002). Information literacy as a catalyst for educational change: a background paper. White Paper prepared for UNESCO, the U.S. National Commission on Libraries and Information Science, and the National Forum on Information Literacy, for use at the Information Literacy Meeting of Experts, Prague, The Czech Republic. http://www.nclis.gov/libinter/infolitconf\&meet/papers/bruce-fullpaper.pdf.

Casas, J. (2003). Modelos de diseño de las TIC. Barcelona: Universitat Oberta de Catalunya.

Castells, M. (1996). The Information Age: Economy, Society and Culture. Vol. 1. The Rise of the Network Society. Oxford: Blackwell Publishers.

Consejo Europeo (2000). Lisbon European Council (23 y 24 de marzo 2000). Presidency Conclusions. Extraído de http://www.consilium.europa.eu/uedocs/cms_data/docs/.../00100-r1.en0.htm.

Ferran, N. \& Virkus, S. (2010). Chapter 5: Information-Related Competences for Teachers and Students in an e-learning Environment. En N. Ferran y J. Minguillón (Eds.), Content Management for eLearning. Nueva York: Springer.

Freedman, T. (2010). The Amazing Web 2.0 Projects Book. Ilford: Terry Freedman Ltd.

Holmes, B. \& Gardner, J. (2006). E-learning: Concepts And Practice. London: Sage Publications.

Johnston, B. and Webber, S. (2003). Information literacy in higher education: a review and case study. Studies.Higher Education, 28 (3), 335-352.

Nielsen, J. (1993). Usability Engineering. San Francisco: Morgan Kaufmann.

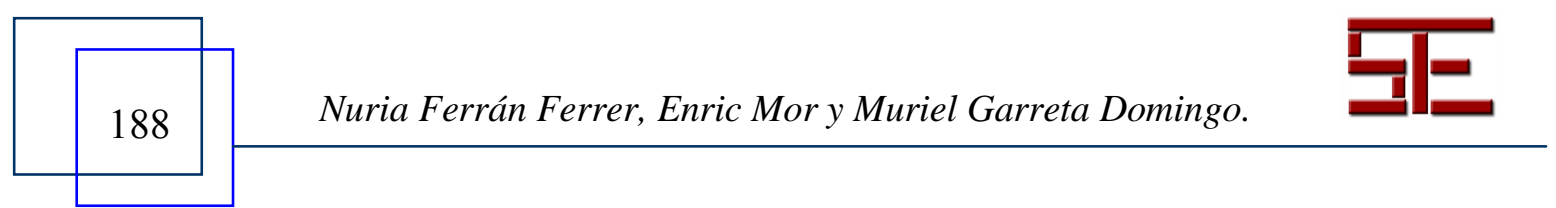




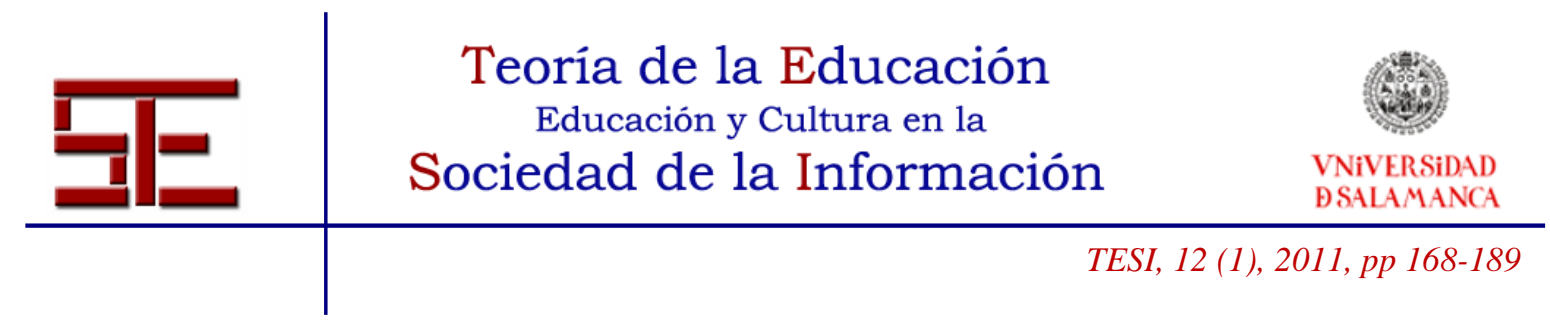

Nielsen, J. \& Molich, R. (1990). Heuristic evaluation of user interfaces. En Proceedings of the SIGCHI conference on Human factors in computing systems: Empowering people. (pp. 249-256). ACM Press.

OECD (2006). Think Scenarios, Rethink Education. Paris: OECD.

Rowlands, I. et. al. (2008). The Google generation: the information behaviour of the researcher of the future. Aslib Proceedings: New Information Perspectives, vol. 60, $\mathrm{N}^{\mathrm{o}}$ 4. 290-310.

SCONUL (2003). Information Support for eLearning: principals and practices. En http://www.sconul.ac.uk/publications/pubs/info_support_elearning.pdf.

Severance, C., Hardin, J. \& Whyte, A. (2008). The coming functionality mashup in personal learning environments. Interactive Learning Environments Journal, vol. 16, $\mathrm{N}^{\mathrm{o}} .1$.

UNESCO (2003). The Prague Declaration: Towards an Information Literate Society.

Para citar el presente artículo puede utilizar la siguiente referencia:

Ferran Ferrer N., Mor E. y Garreta Domingo M. (2011). Optimización del comportamiento informacional mediante el uso de herramientas de bookmarking, en Hernández Serrano, M. J. y Fuentes Agustí, M. (Coords.) La red como recurso de información en educación. Revista Teoría de la Educación: Educación y Cultura en la Sociedad de la Información. Vol. 12, n ${ }^{\circ}$ 1. Universidad de Salamanca, pp. 168-189 [Fecha de consulta: $\mathrm{dd} / \mathrm{mm} / \mathrm{aaa}]$.

http://campus.usal.es/ revistas_trabajo/index.php/revistatesi/article/view/7828/7855

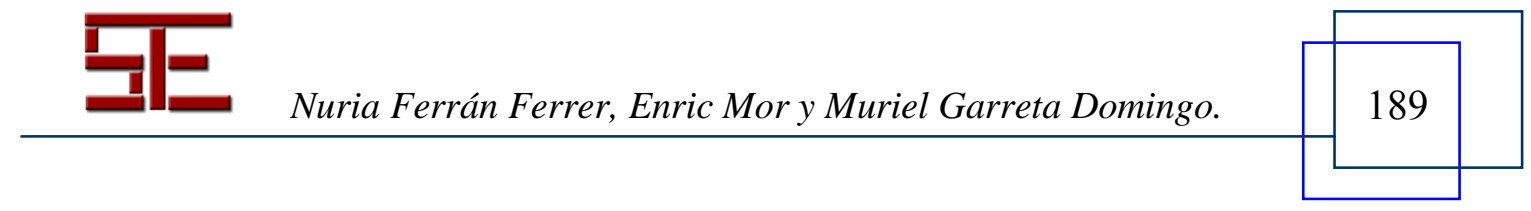

